\title{
JigCell: A New Environment to Simulate the Dynamics of Memory Formation
}

\author{
Onur Alptürk ${ }^{1}$ \\ 1 Istanbul Technical University, Department of Chemistry, 34469, Maslak, Istanbul, Turkey \\ Email: onur.alpturk@itu.edu.tr, Tel: +90-212-2853249, Fax: +90-212-2856386, Address: Istanbul \\ Technical University, Faculty of Science and Letters, Department of Chemistry, Maslak, 34469, \\ Istanbul, Turkey. \\ ORCID NUMBER
}

Onur Alptürk: 0000-0001-6618-4111 


\begin{abstract}
:
Since their initial discovery, long-term potentiation (LTP), and long-term depression (LTD) are accepted as the main biomolecular mechanism that controls memory acquisition. In doing this, both mechanisms are fairly complex and involve specific triggers and many cascades reactions that cross-talk and communicate with others. Thus, they are very complex. To reveal how these mechanisms operate and instruct the brain to remember and forget, one judicious approach is developing the mathematical models of processes. However, this notion requires some basic knowledge regarding ordinary differential equations and writing codes. To this respect, it can be postulated that tools, which can be utilized rather by everyone, would certainly expedite and facilitate the formulation of such models. With this rationale in mind, we demonstrate that JigCell offers the perfect platform to develop such models of LTP. Our choice for this tool stems from the fact that it is designed to simulate complex biological systems in a modular way. Thus, this manuscript is crafted to illustrate how this model was constructed in the JigCell environment and to give an idea of how this tool works.
\end{abstract}

Keywords: Memory formation, Long-term potentiation, Mathematical model, JigCell, Calcium, CaMKII. 


\section{INTRODUCTION}

The question of how we code information in our memory has remained a scientific puzzle, that is inherited from one generation to the next one. However, it should not be interpreted as no progress has been made. On the contrary, so much is known from Hebb's early work. Commonly known as Hebb's rule or cell assembly theory, this theory is summarized as "Cells that fire together wire together". With that, Hebb indeed postulates that activation of pre-and postsynaptic cells strengths the connection between neurons [1]. To communicate more, the synapses of neurons are observed to change, as a result of which their synaptic strength is enhanced. This observation is what we presently know as synaptic plasticity, which depicts the capacity of synapsis to strengthen or weaken in time [2]. Presently, our view of memory formation is dominated by long-term changes in synaptic communication. This effect is accepted to be bidirectional; long-term potentiation (LTP) as a long-lasting increase in signal transmission between two neurons and longterm depression (LTD), in which case the extent of transmission between neurons is weakened. In summary, LTP and LDP are accepted as the biomolecular mechanism that underlies memory acquisition [3].

The structure of how LTP operates starts up with specific triggers. Under the resting condition, the pores of NMDA (N-methyl-D-aspartate) receptors are occupied with $\mathrm{Mg}^{2+}$ ions. Upon depolarization, $\mathrm{Mg}^{2+}$ is removed from the pore, which culminates in the influx of $\mathrm{Na}^{+}, \mathrm{K}^{+}$, and $\mathrm{Ca}^{2+}[4]$. Of them, calcium influx triggers a postsynaptic cascade of reactions that alters the density of AMPA receptors at the post-synapsis. In this process, the frequency in calcium dictates the fate of memory formation by impacting the direction on the alteration of AMPA receptors; highfrequency calcium influx results in LTP, whereas low-frequency calcium influx actuates LTD. Thus, calcium influx has a bidirectional role in deciding whether we remember or we forget [5].

There is no need to mention that in reality, the process of how the brain function is far more perplexing than what is described. For this reason, the way to fully grasp and study all biochemical processes postulates composing mathematical models regarding biochemical events taking place during memory formation. With this motivation, many groups (including ours) have developed models that cover either all or a certain fraction of LTP (for instance, see [6-10]).

Conspicuously, such mathematical models could only be formulated by researchers who are familiar with solving ordinary differential equations or writing codes. To this respect, software that can be utilized by everyone without requiring too much background in mathematics and 
computer science will assuredly expedite progress in this field. For this reason, the JigCell Model Connector was introduced to the scientific community [11]. This software is specially devised to construct molecular network models which are inherently complex. By design, JigCell allows hierarchical model composition; that is to say, a complex network is engineered as a combination of smaller models. In the JigCell environment, these smaller models are named "modules" and each one represents a well-defined fragment of the model. Once the modules are connected through the so-called "interface ports", the overall network is obtained. This way, one can understand how a certain part of the model behaves and interacts with others. From standpoint of user-friendliness, JigCell is very advantageous; the ordinary equations are written through a panel without demanding a code to solve them. Thus, it can be used by anyone.

Given the complexity of the mechanism underlying memory formation, it is reasoned that this software offers the perfect platform to elucidate the dynamics in LTP. To address the applicability of JigCell in this context, a model that covers the Ca2+/CaMKII network is formed as an illustration, with the use of differential equations reported in the literature. Ultimately, this manuscript is crafted to discuss how this model is formed in the JigCell environment and more importantly, to give the reader a feel about the basics of this software.

\section{MATERIAL AND METHODS}

JigCell and COPASI (analysis environment for JigCell) can be downloaded for free from the link given in [12]. In the JigCell environment, the model designed to simulate the Ca2+/CaMKII network is envisioned to harbor two modules; Module 1 and Module 1. In this model, Module 1 relates to the chemistry of calcium, which serves as the well-established initiation signal of LTP. On the other hand, Module 2 deals with the dynamics of CaMKII, a protein kinase known to activate the following downstream reactions in memory formation.

Before discussing the content of each module, this section of the manuscript envisions providing some insight on how this software works. In general, the JigCell environment offers two rationales to implement rules for dynamic changes. The first one is through the "species" menu; the dynamics of reactants and variables (collectively coined as "species") are given through ordinary differential equations (ODE). To illustrate, this rationale was utilized in Module 1, wherein the calcium influx is delineated by the ODE given in the box "expression" (Figure 1). 


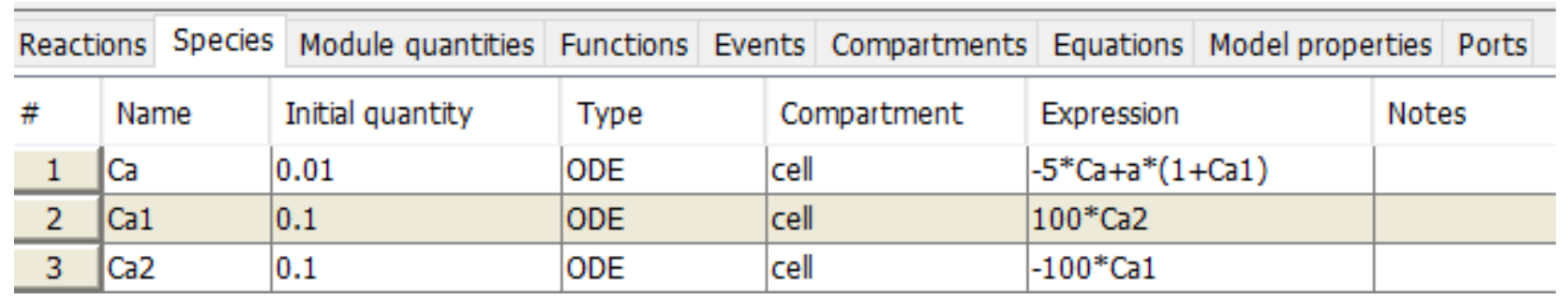

Figure 1. A screenshot of the menu "Species".

The second one involves the representation of these dynamic changes in terms of chemical reactions, as in Module 2. This one is more suited to the cases with multiple species reacting in the form of a cascade (such as phosphorylation/dephosphorylation reaction of CaMKII subunits). For this one, the components of each reaction are predefined in the menu "Functions", wherein the left column is utilized to itemize the components of each reaction in the form of reactants ("MOD") or rate-constants ("VAR"). Subsequently, the right column sets the reaction rules that combine the stoichiometric coefficients, and the rate constants with the reactants (Figure 2).

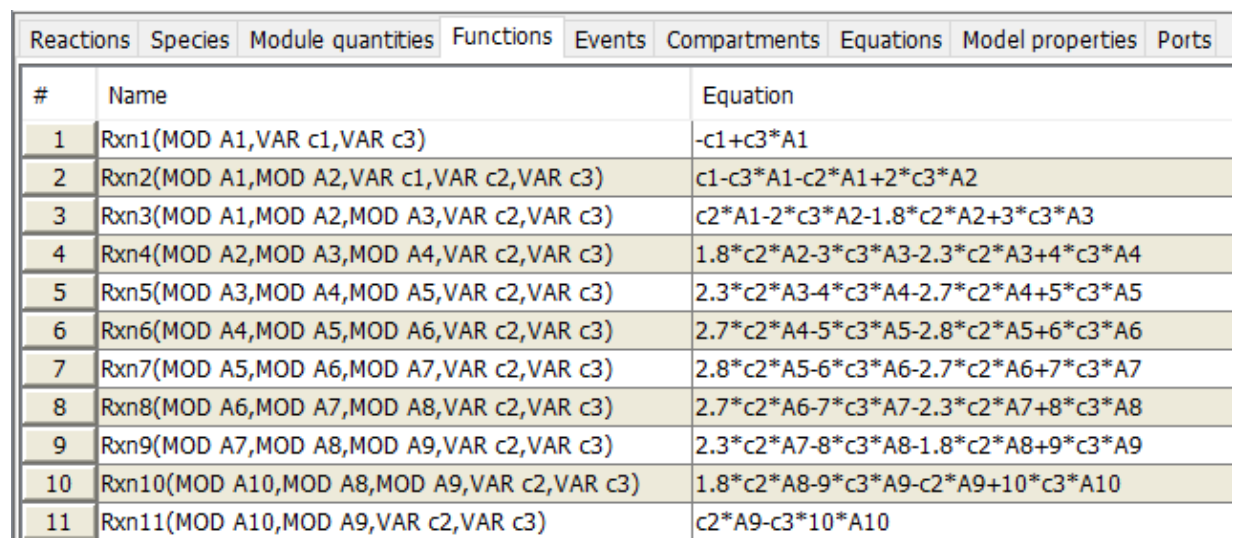

Figure 2. A screenshot of the menu "functions".

With the rate constants and reactants defined once again as "species" (Figure 1), the kinetic law for each reaction is then formulated through the menu "Reactions" (Figure 3). 


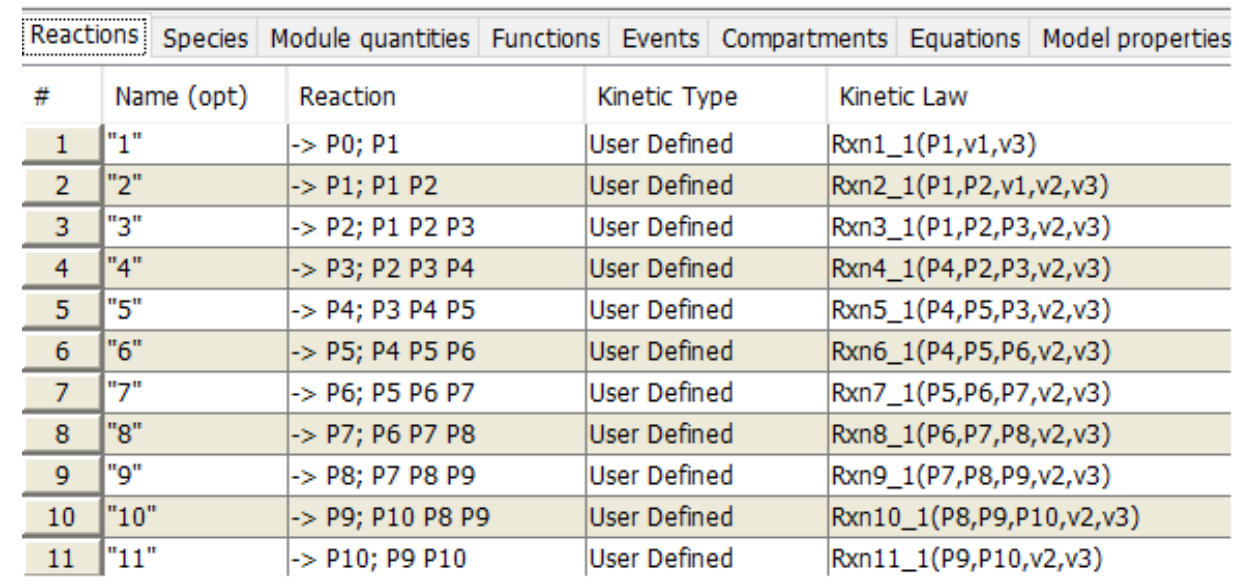

Figure 3. A screenshot of the menu "Reactions".

To obtain the model, Module 1 (Figure 4) and Module 2 are connected through Ca port, whereby the chemical information is conveyed from Module 1 to its partner, Module 2 (as an example, a layout of Module 1 and the model are shown in Figure 4 and 5, respectively). Then, the model is exported with the SBML extension and is imported into the COPASI environment. Finally, the simulation is run from the "Time Course" menu, after its parameters are entered (in this paper, they are: duration is $10 \mathrm{~s}$, intervals is 100 , and interval size is $0.01 \mathrm{~s}$ ). COPASI also has an interface to visualize the results of simulations. As a note, the files of this model are available from GitHub) [13].

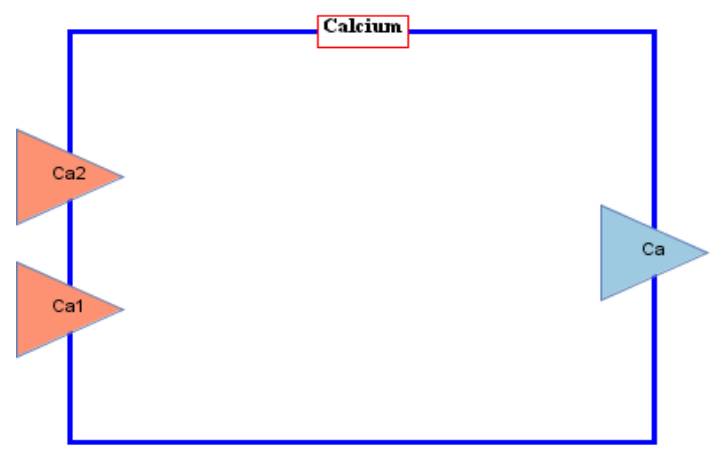

Figure 4. The layout of Module 1. Note that the inputs and the outputs of Module 1 are marked in red and blue, respectively. 


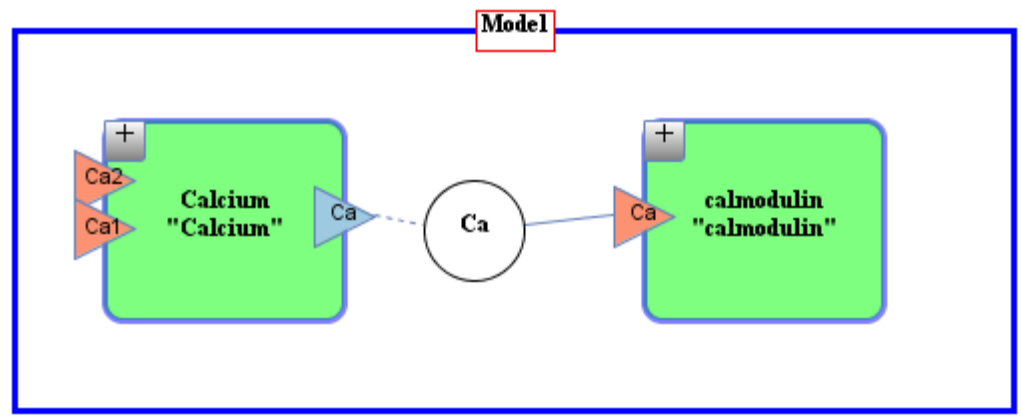

Figure 5. The layout of the model designed to simulate the $\mathrm{Ca}^{2+} / \mathrm{CaMKII}$ network in LTP.

\section{RESULTS AND DISCUSSION}

This section will elaborate on the nature of the modules, as well as the differential equation utilized in each one. Then, the results from the simulation will be discussed.

\subsection{Module 1: Calcium Module}

Serving as the initiation signal for LTP, the calcium module aims at simulating the dynamics of calcium uptake. A general rule, mathematical models solely consider the influx of calcium to post-synaptic neurons as a stimulant to LTP, whilst the roles of calcium from other intracellular sources (such as nuclear calcium) are very much omitted. Hence, calcium $\left(\mathrm{Ca}^{2+}\right)$ in Module 1 stands for synaptic calcium in this manuscript. Of paramount importance is the notion that the model presented hereby doesn't consider any event prior to the calcium entry to post synapsis (for instance, membrane depolarization or displacement of $\mathrm{Mg}^{2+}$ from NMDA receptors). Thus, the oscillatory entry of calcium given in Module1 functions as "the trigger" in this model.

Conventionally, the calcium influx is expressed as an instant elevation, followed by an exponential decay, as tabulated in Equation (1) [14].

$$
\left[\mathrm{Ca}^{2+}\right]=\left[\mathrm{Ca}^{2+}\right]_{\mathrm{rest}}+A \sum_{i=1}^{n} \exp \left(-\frac{i}{f \tau}\right)
$$

where $\left[\mathrm{Ca}^{2+}\right]_{\text {rest }}$ is the resting concentration of calcium, $\mathrm{A}$ is the amplitude of a single $\mathrm{Ca}^{2+}$ pulse, $\mathrm{f}$ is the frequency of excitation, $\tau$ is the relaxation time of $\mathrm{Ca} 2+$ decay, and $\mathrm{n}$ is the number of pulses in the tetanic stimuli (i.g., high-frequency sequence of individual stimulations), with frequency varying from $5 \mathrm{~Hz}$ to $100 \mathrm{~Hz}$. In constructing Module 1, the formulation of equation (1) in the JigCell environment posed a challenge, as such that this software doesn't recognize summation or exponential function. For this reason, equation (1) was redevised as a set of ordinary 
differential equations, wherein equation (2) describes the calcium influx, whilst equations (3), and (4) effectuate characteristic periodicity in the equation (2) with a frequency of $100 \mathrm{~Hz}$ (therein, variables $\mathrm{Ca} 1$ and $\mathrm{Ca} 2$ don't have any biological meaning). The kinetics of calcium influx described by equations (2-4) is shown in Figure 5 (parameters in these equations are described in Table 1).

$$
\begin{gathered}
\frac{d[\mathrm{Ca}](t)}{d t}=-\tau[\mathrm{Ca}](t)+\alpha\left\{1+\left(\mathrm{Ca}_{1}\right)(t)\right\} \\
\frac{d\left(\mathrm{Ca}_{1}\right)(t)}{d t}=\Omega\left(\mathrm{Ca}_{2}\right)(t) \\
\frac{d\left(\mathrm{Ca}_{2}\right)(t)}{d t}=-\Omega\left(\mathrm{Ca}_{1}\right)(t)
\end{gathered}
$$

\begin{tabular}{c|l|c}
\hline \hline Parameters & \multicolumn{1}{|c}{ Description } & Value \\
\hline \hline $\boldsymbol{\alpha}$ & Strength of & $50 \mathrm{M}$ \\
& signal & \\
$\boldsymbol{\Omega}$ & Frequency & $100 \mathrm{~Hz}$ \\
$\boldsymbol{\tau}$ & Time-constant & $5 \mathrm{~s}^{-1}$ \\
\hline \hline
\end{tabular}

Table 1. Parameters in equations 2-4.

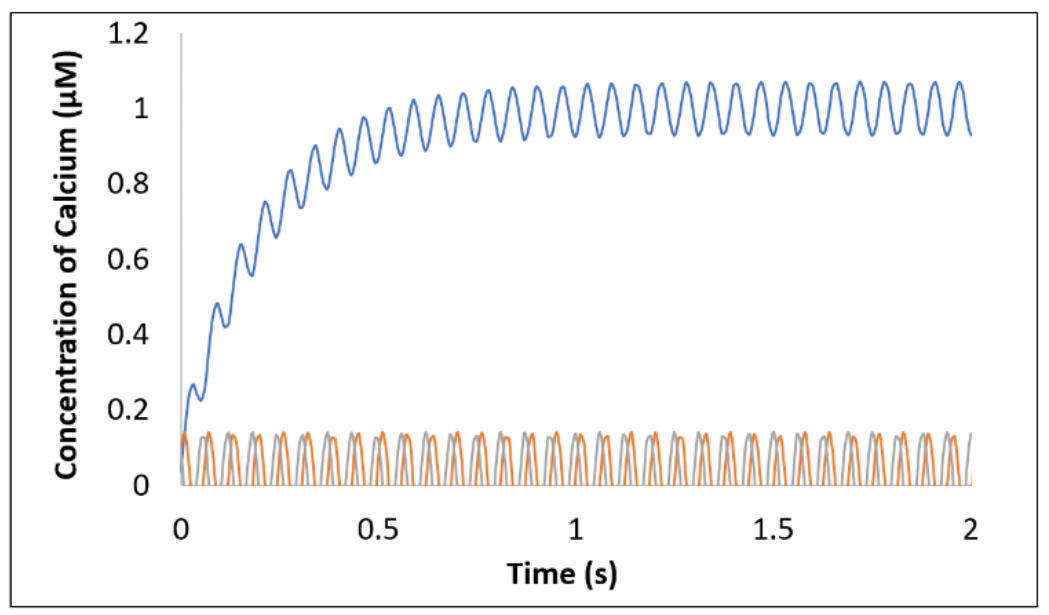

Figure 5. The kinetics of calcium influx defined by the set of equations (2-4) (the basal concentration of calcium is $10 \mathrm{nM}$ and the initial values of $\mathrm{Ca}_{1}$ and $\mathrm{Ca}_{2}$ are 0.1).

Unsurprisingly, this approach fails to reflect the exponential decay in equation 1. To engineer this behavior, the equation (2) was revised to the equation (5), by expressing the strength of the 
signal in the form of a time-dependent variable. In doing this, the prediction was that this way, it would stimulate the calcium influx when initially equal to 50, whereas it would actuate the exponential decay, once set to 0 .

$$
\begin{gathered}
\frac{d[\mathrm{Ca}](t)}{d t}=-\tau[\mathrm{Ca}](t)+\alpha(t)\left\{1+\left(\mathrm{Ca}_{1}\right)(t)\right\} \\
\frac{d\left(\mathrm{Ca}_{1}\right)(t)}{d t}=\Omega\left(\mathrm{Ca}_{2}\right)(t) \\
\frac{d\left(\mathrm{Ca}_{2}\right)(t)}{d t}=-\Omega\left(\mathrm{Ca}_{1}\right)(t) \\
\alpha(t)= \begin{cases}50 & \text { if } 0 \leq t \leq 5 \mathrm{secs}, \\
0 & \text { otherwise. }\end{cases}
\end{gathered}
$$

In the JigCell environment, discontinuity in functions is arranged from the menu "events" that stipulates two entries: "actions" to specify the nature of the triggered event and "delay" to indicate

\begin{tabular}{|c|c|c|c|c|c|c|c|c|c|}
\hline Reactions & ions Species & Module quantities & Functions & Events & Compartments & Equations & \multicolumn{2}{|c|}{ Model properties } & Ports \\
\hline \# & Name (opt) & Trigger expr & ession & Action & Delay & & DlyCalc & Notes & \\
\hline 1 & Calcium trigger & $\mathrm{Ca}>0.04$ & & $a=0$ & 5 & & $\checkmark$ & & \\
\hline 2 & & & & & & & $\square$ & & \\
\hline
\end{tabular}
the trigger time for the event (Figure 6). With this menu, the variable $\alpha$ was programmed to switch from 50 to 0 after 5 seconds, whereupon it would serve as a turn-off signal for the calcium uptake, initiating the exponential decay in the equation (5) (Figure 7).

Figure 6. A screenshot of the menu "events" to introduce an exponential decay in the equation (5).

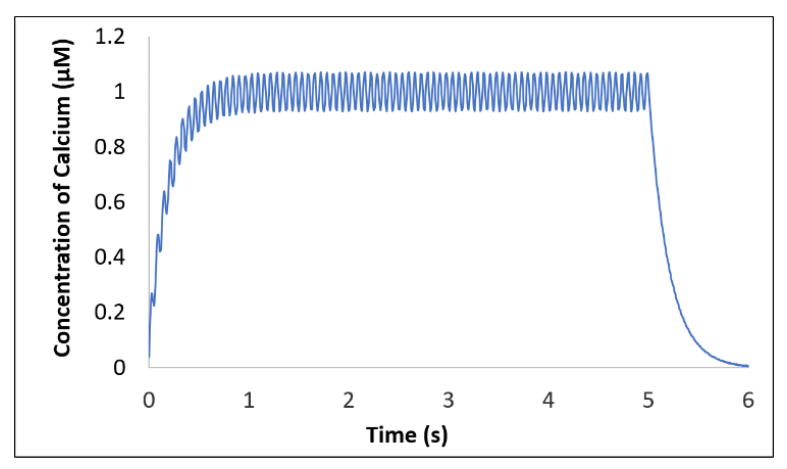

Figure 7. The kinetics of oscillatory calcium influx described by the set of equations (5). 
Once completed, Module 1 is of 2-inputs/1-output configuration, wherein $\mathrm{Ca} 1$ and $\mathrm{Ca} 2$ are the inputs as $\mathrm{Ca}$ is the output that stimulates the phosphorylation of $\mathrm{Ca} 2+/$ calmodulin-dependent protein kinase II (CaMKII) in Module 2 (vide infra).

\subsection{Module 2: Calmodulin Module}

Module 2 is devoted to the chemistry of CaMKII, on the grounds that this kinase is believed to impact the fluctuations of the size and strength of neuronal connection [15]. In the context of LTP, such fluctuations are referred to as "synaptic plasticity", which controls the extent of communication amongst neurons [16,17].

Being a determinant of how we remember, CaMKII is a serine/threonine kinase, which mediates $\mathrm{Ca}^{2+}$-dependent phosphorylation of many neuronal targets. The crystal structure of this enzyme reveals that the holoenzyme is made up of $8-10$ of two subunits, $52-\mathrm{kDa} \alpha$ and $60-\mathrm{kDa} \beta$. These subunits are arranged in the form of two rings, which pivot between an active and inactive conformation [18]. Under basal conditions, the kinase activity of CaMKII is blocked by the binding of the autoinhibitory domain to the catalytic domain. With the calcium transient, calcium/calmodulin $\left(\mathrm{Ca}^{2+} / \mathrm{CaM}\right)$ binds to the autoinhibitory domain and restores the kinase activity of CaMKII by removing it. Later on, this induces autophosphorylation of Thr-286 and phosphorylation of other numerous targets in downstream reactions, which eventually leads up to LTP [19].

Given its significance, many models have been proposed to accurately express and understand how this kinase responds to intracellular calcium [20-23]. Amongst them, the one developed by Zhabotinsky is particularly intriguing as it reported a rather simple one to elucidate the mechanism of autophosphorylation of CaMKII in the presence of a calcium-dependent phosphatase [20]. Therefore, this model was of choice for the design of Module 2.

To make the mechanism of this enzyme more intelligible, Zhabotinsky made certain assumptions. For instance, this model fully ignores any difference between $\alpha$ and $\beta$ subunits, treating CaMKII as a decamer of a single type of subunits. Then, the activation of subunits by calcium obeys the Hill equation, and the binding of $\mathrm{Ca}^{2+} / \mathrm{CaM}$ and the phosphatase to a subunit is independent of the phosphorylation state of other subunits.

Quite naturally, this manuscript will manifest certain key aspects of this model; for further details, the reader should refer to the original paper. To begin with, CaMKII is portrayed as a single ring of these subunits, in which the cascade of phosphorylation propagates in one direction, as 
originally proposed by Hanson and Schulman [24]. In the initiation step of phosphorylation reactions, $(\mathrm{Ca} 2+) 4 \mathrm{CaM}$ binds to two neighbor subunits. Then, the first subunit phosphorylates the second one in the clockwise direction, as shown by the reactions (9-12).

$$
\begin{gathered}
4 \mathrm{Ca}^{2+}+\mathrm{CaM} \rightleftharpoons \mathrm{C} \\
\mathrm{P}_{0}+\mathrm{C} \rightleftharpoons \mathrm{P} \mathrm{C} \\
\mathrm{P}_{0} \mathrm{C}+\mathrm{C} \rightleftharpoons \mathrm{P}_{0} \mathrm{C}_{2} \\
\mathrm{P}_{0} \mathrm{C}_{2} \rightarrow \mathrm{P}_{1} \mathrm{C}_{2}
\end{gathered}
$$

where C represents $\left(\mathrm{Ca}^{2+}\right)_{4} \mathrm{CaM}$ complex. P0 and P1 are unphosphorylated and 1-fold phosphorylated haloenzyme, respectively.

Next is the incorporation of the dephosphatase activity. There are four protein phosphatases known to act upon CaMKII-P [25], however; this model solely deals with PP1, given that this is the only protein that dephosphorylates CaMKII in the postsynaptic densities [25,26]. In this regard, it was demonstrated that the activity of PP1 is regulated by $\mathrm{Ca}^{2+} / \mathrm{CaM}$ via inhibitor 1 , calcineurin (CaN), and cAMP-dependent protein kinase (PKA). Of note, inhibitor 1 (I1) is phosphorylated by PKA and dephosphorylated by Calcineurin; once phosphorylated, I1 deactivates PP1 [27,28]. In terms of LPT, I1 is of paramount significance as its role renders this element a regulatory factor that links the calcium influx to synaptic plasticity [29]. Overall, the model of autophosphorylation of CaMKII in the presence of PP1 is given with the following equations (the constants $\mathrm{c} 1-\mathrm{c} 4$ and the parameters therein are defined in Table 2, and Table 3, respectively).

$$
\begin{gathered}
\frac{d P_{0}(t)}{d t}=-v_{1}+v_{3} P_{1} \\
\frac{d P_{1}(t)}{d t}=v_{1}-v_{3} P_{1}-v_{2} P_{1}+c_{1} v_{3} P_{2} \\
\frac{d P_{2}(t)}{d t}=v_{2} P_{1}-c_{1} v_{3} P_{2}-c_{2} v_{2} P_{2}+c_{3} v_{3} P_{3} \\
\frac{d P_{3}(t)}{d t}=c_{1} v_{2} P_{2}-c_{2} v_{3} P_{3}-c_{3} v_{2} P_{3}+c_{4} v_{3} P_{4} \\
\frac{d P_{4}(t)}{d t}=c_{1} v_{2} P_{3}-c_{2} v_{3} P_{4}-c_{3} v_{2} P_{4}+c_{4} v_{3} P_{5} \\
\frac{d P_{5}(t)}{d t}=c_{1} v_{2} P_{4}-c_{2} v_{3} P_{5}-c_{3} v_{2} P_{5}+c_{4} v_{3} P_{6}
\end{gathered}
$$




$$
\begin{gathered}
\frac{d P_{6}(t)}{d t}=c_{1} v_{2} P_{5}-c_{2} v_{3} P_{6}-c_{3} v_{2} P_{6}+c_{4} v_{3} P_{7} \\
\frac{d P_{7}(t)}{d t}=c_{1} v_{2} P_{6}-c_{2} v_{3} P_{7}-c_{3} v_{2} P_{7}+c_{4} v_{3} P_{8} \\
\frac{d P_{8}(t)}{d t}=c_{1} v_{2} P_{7}-c_{2} v_{3} P_{8}-c_{3} v_{2} P_{8}+c_{4} v_{3} P_{9} \\
\frac{d P_{9}(t)}{d t}=c_{1} v_{2} P_{8}-c_{2} v_{3} P_{9}-v_{2} P_{9}+c_{3} v_{3} P_{10} \\
\frac{d P_{10}(t)}{d t}=v_{2} P_{9}-c_{1} v_{3} P_{10} \\
\frac{d e_{p}(t)}{d t}=-k_{3} I e_{p}+k_{4}\left(e_{p 0}-e_{p}\right) \\
\frac{d I(t)}{d t}=-k_{3} I e_{p}+k_{4}\left(e_{p 0}-e_{p}\right)+v_{P K A} I_{0} \\
-\frac{V_{C a N}\left([C a] / K_{H 2}\right)^{3} I}{1+\left([C a] / K_{H 2}\right)^{3}}
\end{gathered}
$$

where the rate constants $\mathrm{v} 1, \mathrm{v} 2$, and $\mathrm{v} 3$ are:

$$
\begin{gathered}
v_{1}=\frac{10 k_{1}\left([C a] / K_{H 1}\right)^{8} P_{0}}{\left(1+\left([C a] / K_{H 1}\right)^{4}\right)^{2}} \\
v_{2}=\frac{k_{1}\left([C a] / K_{H 1}\right)^{4} P_{1}}{\left(1+\left([C a] / K_{H 1}\right)^{4}\right.} \\
v_{3}=\frac{k_{2} e_{p}}{K_{M}+\sum_{i=1}^{10} i P_{i}}
\end{gathered}
$$




\begin{tabular}{c|cccc}
\hline \hline Equation & $\mathbf{c}_{\mathbf{1}}$ & $\mathbf{c}_{\mathbf{2}}$ & $\mathbf{c}_{\mathbf{3}}$ & $\mathbf{c}_{\mathbf{4}}$ \\
\hline \hline $\mathbf{2 1}$ & 2 & - & - & - \\
$\mathbf{2 2}$ & 2 & 1.8 & 3 & - \\
$\mathbf{2 3}$ & 1.8 & 3 & 2.3 & 4 \\
$\mathbf{2 4}$ & 2.3 & 4 & 2.7 & 5 \\
$\mathbf{2 5}$ & 2.7 & 5 & 2.8 & 6 \\
$\mathbf{2 6}$ & 2.8 & 6 & 2.7 & 7 \\
$\mathbf{2 7}$ & 2.7 & 7 & 2.3 & 8 \\
$\mathbf{2 8}$ & 2.3 & 8 & 1.8 & 9 \\
$\mathbf{2 9}$ & 1.8 & 9 & 10 & - \\
$\mathbf{3 0}$ & 10 & - & - & - \\
\hline \hline
\end{tabular}

Table 2. The constants $c_{1}-c_{4}$ in equations (13-23).

\subsection{The Model}

The results of the simulation indicate that this cascade proceeds as a response to calcium, which concurrently orchestrates phosphorylation and dephosphorylation reactions. In this regard, it is observed that when $\mathrm{Ca} 2+$ firstly triggers $\mathrm{n}$-fold phosphorylation reactions of CaMKII subunits, as a result of which unphosphorylated holoenzyme (P0) is fully consumed and evokes the synthesis of holoenzyme with a higher degree of phosphorylation. Mechanistically, the kinetics of each reaction exhibit bell-curve-like behavior in that each signal sequentially reaches its maximum, and then, undergoes "dephosphorylation", forming the cascade of "n-fold dephosphorylation". Thus, this model consists of two series of reactions merged to form a cascade; $\mathrm{n}$-fold phosphorylation reactions $\left(\mathrm{P}_{0} \rightarrow \mathrm{P}_{1} \rightarrow \mathrm{P}_{2} \ldots \mathrm{P}_{8} \rightarrow \mathrm{P}_{9}\right)$, and $\mathrm{n}$-fold dephosphorylation reactions $\left(\mathrm{P}_{10} \rightarrow \mathrm{P}_{9} \rightarrow \mathrm{P}_{8} \ldots\right.$ $\left.\mathrm{P}_{1} \rightarrow \mathrm{P}_{0}\right)$.

From standpoint of LTP, the phosphorylation of subunits and kinase activity in post-synapsis come to mean the signal, which is essential to catalyze the other downstream events in LTP. After fulfilling its role, this signal is shut down through dephosphorylation of holoenzymes, as discussed before. However, it is worth noting that according to this model, the concentration of subunits doesn't fully return to their initial values, indicating partial dephosphorylation, rather than complete dephosphorylation. One may speculate that this stems from the dynamic of phosphatase and inhibitor-1 cycle, which should govern the turn-off signal. To elaborate, calcium accumulating in the post-synapsis yields the concurrent synthesis of free inhibitor 1 (I1, Figure 9) and phosphatase (Ep, Figure 10). This results in a temporary inhibition of phosphatase activity, sustaining the n-fold phosphorylation cascade, as seen in Figure 8. When the calcium influx begins 
to decay at $\mathrm{t}=5 \mathrm{~s}$, however; one would expect that the balance in Ep/ I1 should have favored phosphatase activity, whereby the subunits of CaMKII are thoroughly dephosphorylated. In contrast, it appears that the extent of phosphatase activity tends to decrease beyond $\mathrm{t}=5 \mathrm{~s}$. Thus, the lack of sufficient phosphatase activity may be responsible for CaMKII subunits preserving their phosphorylated states and not fully turning the kinase activity off at $\mathrm{t}>5 \mathrm{~s}$.

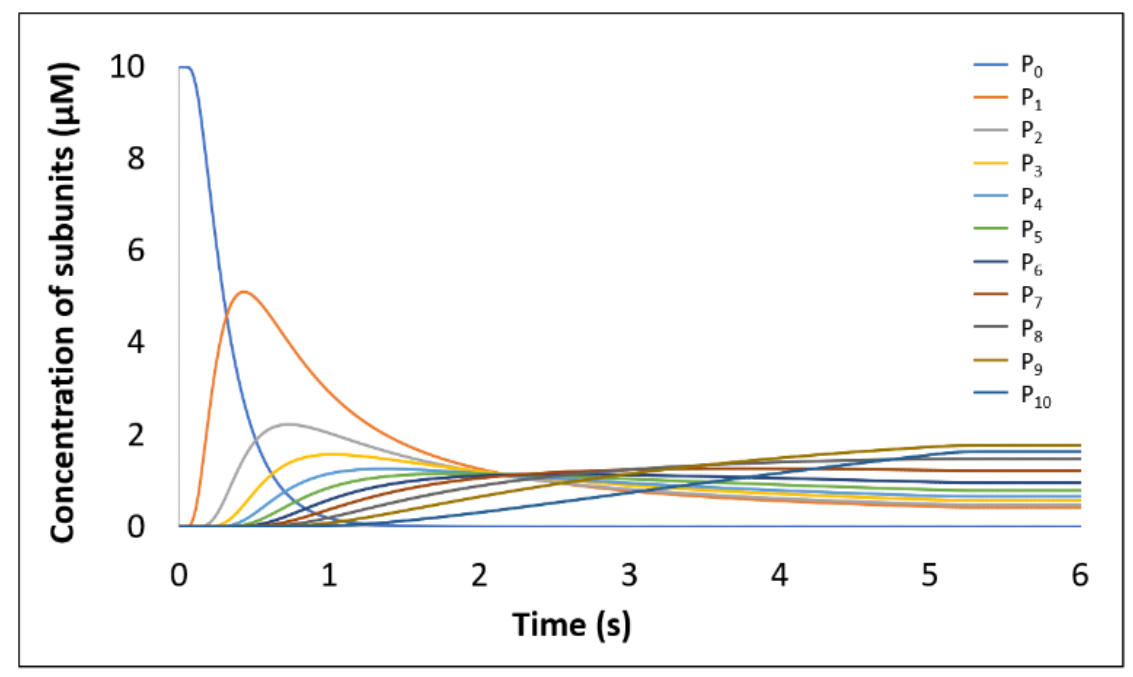

Figure 8. Kinetics of CaMKII subunit phosphorylation in response to calcium influx (the initial concentration of $\mathrm{P}_{0}$ is $10 \mu \mathrm{m}$ whereas that of other subunits are considered to be $0 . \mathrm{K}_{\mathrm{m}}$ and $\mathrm{KH}_{2}$ are $10 \mu \mathrm{M}, 0.6 \mu \mathrm{M}$, respectively) [20].

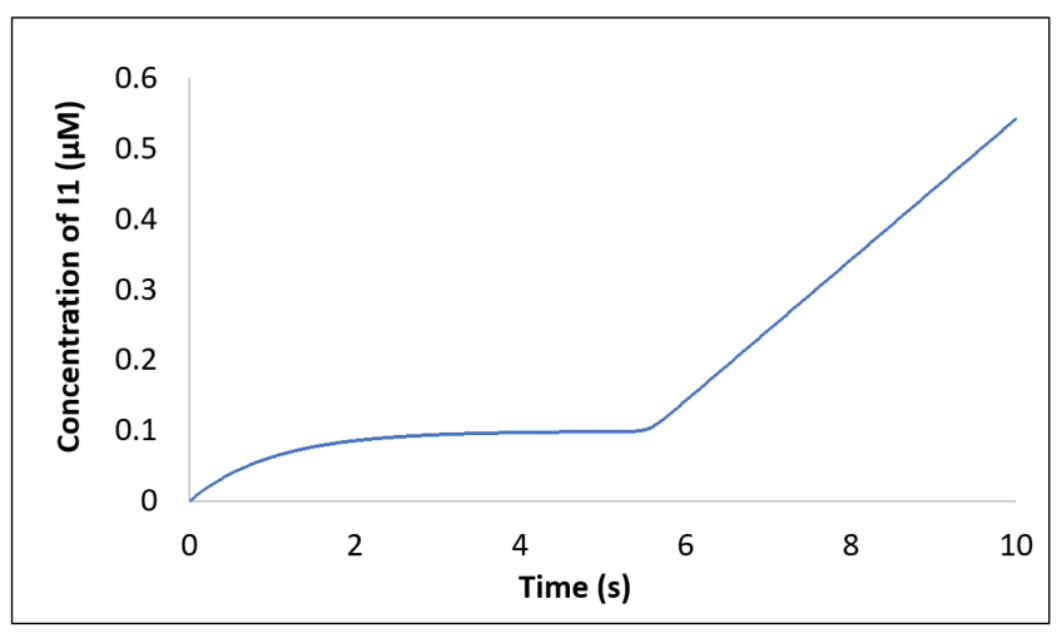

Figure 9. Kinetics of I1 in the response to calcium influx. 


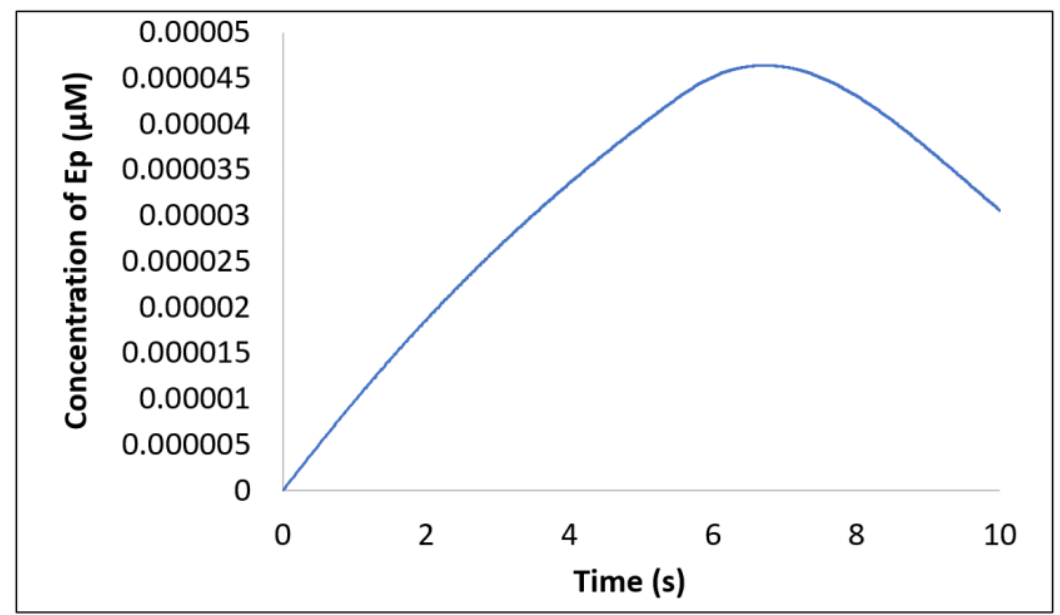

Figure 10. Kinetics of $E_{p}$ in the response to calcium influx $\left(E_{p 0}\right.$ is $\left.0.6 \mu M\right)$ [20].

\begin{tabular}{|c|c|c|c|c|}
\hline Parameter & Description & Value & Unit & Reference \\
\hline $\mathbf{e}_{\mathbf{k}}$ & Total concentration of CaMKII & $0.1-30$ & $\mu \mathrm{M}$ & 25 \\
\hline epo & $\begin{array}{l}\text { Total concentration of protein } \\
\text { phosphatase }\end{array}$ & $0.01-1.2$ & $\mu \mathrm{M}$ & 20 \\
\hline $\mathbf{I}_{\mathbf{0}}$ & Concentration of free inhibitor 1 & $0.0,0.1$ & $\mu \mathrm{M}$ & 20 \\
\hline $\mathbf{V}_{\text {CaN }}$ & $\begin{array}{l}\text { Activity of calcineurin divided by its } \\
\text { Michaelis constant }\end{array}$ & 1.0 & $\mathrm{~s}^{-1}$ & 20 \\
\hline VPKA & $\begin{array}{l}\text { Activity of PKA divided by its } \\
\text { Michaelis constant }\end{array}$ & 1.0 & $\mathrm{~s}^{-1}$ & 20 \\
\hline $\mathbf{K}_{\mathbf{M}}$ & $\begin{array}{l}\text { The Michaelis constant of protein } \\
\text { phosphatase }\end{array}$ & $0.4-20$ & $\mu \mathrm{M}$ & 20 \\
\hline$K_{H 1}$ & $\begin{array}{l}\text { The } \mathrm{Ca}^{2+} \text { activation Hill constant of } \\
\text { CaMKII }\end{array}$ & 4.0 & $\mu \mathrm{M}$ & {$[30,31]$} \\
\hline $\mathbf{K}_{\mathbf{H} 2}$ & $\begin{array}{l}\text { The } \mathrm{Ca}^{2+} \text { activation Hill constant of } \\
\text { calcineurin }\end{array}$ & $0.3-1.4$ & $\mu \mathrm{M}$ & 32 \\
\hline $\mathbf{k}_{1}$ & $\begin{array}{l}\text { The catalytic constant of } \\
\text { autophosphorylation }\end{array}$ & 0.5 & $\mathrm{~s}^{-1}$ & 33 \\
\hline k2 & $\begin{array}{l}\text { The catalytic constant of protein } \\
\text { phosphatase }\end{array}$ & 2.0 & $\mathrm{~s}^{-1}$ & 20 \\
\hline k3 & $\begin{array}{l}\text { The association rate constant of the } \\
\text { PP1 z I1P complex }\end{array}$ & 1.0 & $\mu \mathrm{M}$ & {$[20,28]$} \\
\hline $\mathbf{k} 4$ & $\begin{array}{l}\text { The dissociation rate constant of the } \\
\text { PP1 } \bullet 11 \text { P complex }\end{array}$ & $1 \times 10^{-3}$ & $\mathrm{~s}^{-1}$ & {$[20,28]$} \\
\hline
\end{tabular}

Table 3. Parameters of Module-2. 


\section{CONCLUSION}

In summary, the results reveal that the model formulated hereby is an avenue to study the $\mathrm{Ca}^{2+} / \mathrm{CaMKII}$ network in the JigCell environment. Needless to say, this model will serve as a theoretical experimental environment, in which one can alter the parameter and effortlessly elucidate its impact on the process. Of course, the next step should be the validation and finetuning of such models through some experimental results; once done, a better understanding of memory formation will be obtained. In long term, more accurate models that are composed this way will aid the scientific community to understand how we learn and but also, how our brain fails us, as in neurodegenerative diseases.

One final remark concerns JigCell and COPASI. To this date, these tools have been utilized to analyze many complex systems (for instance, see [34]). However, their use in the context of LTP has not been precedented, to the best of our knowledge. Thus, this study will expand the repertoire of their applications. Within this scope, the development of new and more elaborative models is currently in progress, in our laboratories (see: https://onuralpturk8.wix site.com/projectcerebra).

\section{ACKNOWLEDGEMENT}

This study was carried out in the Chemistry Department of Istanbul Technical University (Bioorganic and Biochemistry Laboratory). The author kindly acknowledges Prof. Dr. Neslihan S. Şengör for the valuable suggestions she provided throughout the preparation of this manuscript.

\section{REFERENCES}

[1] Hebb, D. O. (1949). The Organization of Behavior. New York, NY: Wiley \& Sons.

[2] Hughes, J. R. (1958). Post-tetanic potentiation. Physiological Reviews. 38 (1): 91-113

[3] Bliss, T. V., \& Lømo, T. (1973). Long-lasting potentiation of synaptic transmission in the dentate area of the anaesthetized rabbit following stimulation of the perforant path. The Journal of physiology, 232(2), 331-356.Baltaci, S. B., Mogulkoc, R., \& Baltaci, A. K. (2019). Molecular mechanisms of early and late LTP. Neurochemical Research, 44(2), 281-296.

[4] Baltaci, S. B., Mogulkoc, R., \& Baltaci, A. K. (2019). Molecular mechanisms of early and late LTP. Neurochemical Research, 44(2), 281-296. 
[5] He, Y., Kulasiri, D., \& Samarasinghe, S. (2016). Modelling bidirectional modulations in synaptic plasticity: A biochemical pathway model to understand the emergence of long term potentiation (LTP) and long term depression (LTD). Journal of Theoretical Biology, 403, 159-177.

[6] Alptürk, O., \& Şengör, N. S. (2019). A Model for the Effect of Glia on the Communication Amongst Neurons. 2019 27th Signal Processing and Communications Applications Conference (SIU), pp. 1-4.

[7] Lisman, J. E., \& Zhabotinsky, A. M. (2001). A model of synaptic memory: a CaMKII/PP1 switch that potentiates transmission by organizing an AMPA receptor anchoring assembly. Neuron, 31(2), 191-201.

[8] Tewari, S., \& Majumdar, K. (2012). A mathematical model for astrocytes mediated LTP at single hippocampal synapses. Journal of computational neuroscience, 33(2), 341-370.

[9] Ohadi, D., Schmitt, D. L., Calabrese, B., Halpain, S., Zhang, J., \& Rangamani, P. (2019). Computational modeling reveals frequency modulation of calcium-cAMP/PKA pathway in dendritic spines. Biophysical Journal, 117(10), 1963-1980.

[10] Smolen, P., Baxter, D. A., \& Byrne, J. H. (2006). A model of the roles of essential kinases in the induction and expression of late long-term potentiation. Biophysical Journal, 90(8), 2760-2775.

Jones Jr, T. C., Hoops, S., Watson, L. T., Palmisano, A., Tyson, J. J., \& Shaffer, C. A.

[11] (2018). JigCell Model Connector: building large molecular network models from components. Simulation, 94(11), 993-1008.

[12] http://copasi.org/Projects/JigCell_Model_Connector/

[13] https://github.com/oalptu1/Cerebra.git

He, Y., Kulasiri, D., \& Samarasinghe, S. (2016). Modelling bidirectional modulations in

[14] synaptic plasticity: A biochemical pathway model to understand the emergence of long term potentiation (LTP) and long term depression (LTD). Journal of Theoretical Biology, 403, 159-177.

[15] Zalcman, G., Federman, N., \& Romano, A. (2018). CaMKII isoforms in learning and memory: localization and function. Frontiers in Molecular Neuroscience, 11, 445.

[16] Kennedy, M. B. (2016). Synaptic signaling in learning and memory. Cold Spring Harbor perspectives in Biology, 8(2), a016824. 
[17] Citri, A., \& Malenka, R. C. (2008). Synaptic plasticity: multiple forms, functions, and mechanisms. Neuropsychopharmacology, 33(1), 18-41.

Hoelz, A., Nairn, A. C., \& Kuriyan, J. (2003). Crystal structure of a tetradecameric

[18] assembly of the association domain of $\mathrm{Ca} 2+/$ calmodulin-dependent kinase II. Molecular Cell, 11(5), 1241-1251.

[19] Magupalli, V. G., Mochida, S., Yan, J., Jiang, X., Westenbroek, R. E., Nairn, A. C., Scheuer, T., \& Catterall, W. A. (2013). Ca2+-independent activation of $\mathrm{Ca} 2+/$ calmodulin-dependent protein kinase II bound to the $\mathrm{C}$-terminal domain of $\mathrm{CaV} 2$. 1 calcium channels. Journal of Biological Chemistry, 288(7), 4637-4648.

[20] Zhabotinsky, A. M. (2000). Bistability in the $\mathrm{Ca} 2+/$ calmodulin-dependent protein kinasephosphatase system. Biophysical Journal, 79(5), 2211-2221.

[21] Pharris, M. C., Patel, N. M., VanDyk, T. G., Bartol, T. M., Sejnowski, T. J., Kennedy, M. B., Stefan, M. I., \& Kinzer-Ursem, T. L. (2019). A multi-state model of the CaMKII dodecamer suggests a role for calmodulin in maintenance of autophosphorylation. PLoS Computational Biology, 15(12), e1006941.

Lisman, J. E., \& Goldring, M. A. (1988). Feasibility of long-term storage of graded

[22] information by the $\mathrm{Ca} 2+/$ calmodulin-dependent protein kinase molecules of the postsynaptic density. Proceedings of the National Academy of Sciences, 85(14), 53205324.

[23] Michelson, S., \& Schulman, H. (1994). CaM kinase: a model for its activation and dynamics. Journal of Theoretical Biology, 171(3), 281-290.

[24] Hanson, P. I., \& Schulman, H. (1992). Neuronal Ca2+/calmodulin-dependent protein kinases. Annual Review of Biochemistry, 61(1), 559-601.

Strack, S., Choi, S., Lovinger, D. M., \& Colbran, R. J. (1997). Translocation of

[25] autophosphorylated calcium/calmodulin-dependent protein kinase II to the postsynaptic density. Journal of Biological Chemistry, 272(21), 13467-13470.

[26] Yoshimura, Y., Sogawa, Y., \& Yamauchi, T. (1999). Protein phosphatase 1 is involved in the dissociation of $\mathrm{Ca} 2+/$ calmodulin-dependent protein kinase II from postsynaptic densities. FEBS Letters, 446(2-3), 239-242.

[27] Shenolikar, S., \& Nairn, A. C. (1991). Protein phosphatases—recent progress. Adv. Sec. Mess. Phosphoprot. Res. 23:1-121. 
[28] Endo, S., Zhou, X., Connor, J., Wang, B., \& Shenolikar, S. (1996). Multiple structural elements define the specificity of recombinant human inhibitor-1 as a protein phosphatase-1 inhibitor. Biochemistry, 35(16), 5220-5228.

[29] Allen, P. B., Hvalby, Ø., Jensen, V., Errington, M. L., Ramsay, M., Chaudhry, F. A., Bliss, T. V. P., Storm-Mathisen, J., Morris, R. G. M., Andersen, P., \& Greengard, P. (2000). Protein phosphatase-1 regulation in the induction of long-term potentiation: heterogeneous molecular mechanisms. Journal of Neuroscience, 20(10), 3537-3543.

[30] Fährmann, M., Möhlig, M., Schatz, H., \& Pfeiffer, A. (1998). Purification and characterization of a $\mathrm{Ca} 2+/$ calmodulin-dependent protein kinase II from hog gastric mucosa using a protein-protein affinity chromatographic technique. European Journal of Biochemistry, 255(2), 516-525.

[31] De Koninck, P., \& Schulman, H. (1998). Sensitivity of CaM kinase II to the frequency of $\mathrm{Ca} 2+$ oscillations. Science, 279(5348), 227-230.

[32] Stemmer, P. M., \& Klee, C. B. (1994). Dual calcium ion regulation of calcineurin by calmodulin and calcineurin B. Biochemistry, 33(22), 6859-6866.

[33] Hanson, P. I., Meyer, T., Stryer, L., \& Schulman, H. (1994). Dual role of calmodulin in autophosphorylation of multifunctional CaM kinase may underlie decoding of calcium signals. Neuron, 12(5), 943-956.

[34] http://copasi.org/Research/ 